\title{
A Dual Effect of Au-Nanoparticles on Nucleic Acid Cholesteric Liquid-Crystalline Particles
}

\author{
Yuri Mikhailovich Yevdokimov ${ }^{1 *}$, Sergey Gennadevich Skuridin ${ }^{1}$, Victor Ivanovich Salyanov ${ }^{1}$, \\ Vladimir Ivanovich Popenko ${ }^{1}$, Victor Moiseevich Rudoy', Olga Vadimovna Dement'eva², \\ Eleonora Vladimirovna Shtykova ${ }^{3}$
}

${ }^{1}$ Engelhardt Institute of Molecular Biology of the Russian Academy of Sciences, Moscow, Russia; ${ }^{2}$ Frumkin Institute of Physical
Chemistry and Electrochemistry of the Russian Academy of Sciences, Moscow, Russia; ${ }^{3}$ Shubnikov Institute of Crystallography of
the Russian Academy of Sciences, Moscow, Russia.
Email: ${ }^{*}$ yevdokim@eimb.ru

Received June 21 $1^{\text {st }}, 2011$; revised July 26 $6^{\text {th }}, 2011$; accepted August $15^{\text {th }}, 2011$.

\begin{abstract}
Au-nanoparticles (size about $2 \mathrm{~nm}$, but not 5 or $15 \mathrm{~nm}$ ) are capable of effectively incorporating into quasinematic layers of particles of cholesteric liquid-crystalline dispersion formed by double-stranded nucleic acid molecules of various families (DNA and poly(I)xpoly(C)). This Au-size-dependent process is accompanied by a decrease in amplitudes of abnormal bands in the CD spectra specific to initial cholesteric liquid-crystalline dispersions and simultaneously by an appearance of plasmon resonance band in visible absorption spectrum. The study of properties of particles of cholesteric liquid-crystalline dispersion treated with Au-nanoparticles by means of various physico-chemical methods demonstrates that incorporation of Au-nanoparticles into quasinematic layers of these particles results in two effects: i) it facilitates reorganization of the spatial cholesteric structure of particles, and ii) it induces the formation of Au-clusters in the content of particles. It is not excluded that these effects represent a possible reason for genotoxicity of Au-nanoparticles.
\end{abstract}

Keywords: Double-Stranded Nucleic Acids, Gold Nanoparticles, Nucleic Acid Liquid-Crystalline Dispersions, Circular Dichroism, Structure of Lyotropic Liquid Crystals of (Bio)Polymers, Genotoxity of Nanoparticles

\section{Introduction}

Understanding how nanomaterials affect live-cells functions is the principal aim of nanobiotechnology [1]. Although applications of various nanomaterials (nanoparticles or quantum dots) have shown promising potential for medicine, the fundamental interactions and effects of nanomaterials in living systems for the most part remain unknown. This depends mainly on the solution of two problems: 1) there are many nanomaterials with strongly different properties; 2) an adequate, "coordinated" system to test nanomaterials is not elaborated. For instance, it was suggested that biological effect of Au-nanoparticles on spatially organized double-stranded (ds) DNA structures in vitro and in vivo is similar to the effect of mutagenic molecules $[2,3]$. One can stress that the interaction of metal nanoparticles with native single- or double-stranded nucleic acid molecules is a well-known phenomenon [4]. Although, the cytotoxicity of Au-nanoparticles in vitro and in vivo has been examined by se- veral research groups, no general conclusion can be drawn at present [5-8]. It is connected (maybe) to the fact that the functioning of the nanomaterials in living cells was tested in quite different biological test-systems and the estimation of the mechanism (or mechanisms) of this process is a complicated problem $[9,10]$. This means that great importance is attached to creation of simple model systems to test the effects of nanomaterials under reasonable conditions. Despite the fact that in vivo conditions are different from in vitro, one of these model system is the so-called "cholesteric liquid crystals" and "cholesteric liquid-crystalline dispersions" of ds DNA molecules [11]. Indeed, the physico-chemical properties of ds DNA cholesteric liquid-crystalline dispersions (CLCDs) reflect some properties of these macromolecules in biological objects such as chromosomes of primitive organisms (for instance, chromosomes of the Dinoflagellate, etc.) and DNA containing viruses [12]. Hence, this test-system is not only of nanotechnological, 
but of biotechnological interest. Here one can add, that due to the high local concentration of the ds DNA molecules and their ordered arrangement in particles of CLCD, the low-molecular-mass chemical and biologically active compounds quickly diffuse into the content of particles of CLCD and interact with ds DNA molecules forming different complexes [11]. This process induces an appearance peculiarities (specific for these compounds) in the circular (CD) spectra [13]. This means that every particles of the CLCD was considered not only as an effective "adsorber" but as a sensing unit [14] for the low-molecular-mass compounds as well.

However, to the best of our knowledge, the interaction of nanoparticles added to solutions containing particles of CLCDs (PCLCD) formed by ds DNA molecules has not been investigated so far.

The goal of this work is the study of the effects induced by Au-nanoparticles in the DNA PCLCDs using various physico-chemical methods.

\section{Materials and Methods}

Three colloidal gold solutions (hydrosols) with spherical nanoparticles of different sizes were used in our study. The spherical Au-nanoparticles were synthesized according to previously reported methods [13-15]. The first hydrosol was prepared by Turkevich et al. [15] method and contained particles with average diameter of about $15 \mathrm{~nm}$. The second one, with Au-nanoparticles of $5.5 \mathrm{~nm}$ in size, was synthesized according to Natan et al. [16]. Finally, hydrosol containing quasi-metallic Au-nanoparticles with sizes of $2-3 \mathrm{~nm}$ was synthesized as described in [17]. The average size of $\mathrm{Au}$ nanoparticles in stock preparations was estimated by means of dynamic light scattering and by electron microscopy. The particle number concentration was equal to $10^{12} ; 10^{13}$; and $10^{15} \mathrm{~cm}^{-3}$, respectively. Stock Au-nanoparticle solutions were stored in dark in refrigerator at $4^{\circ} \mathrm{C}$ and used 2.5 month after their synthesis.

Calf thymus ds DNA preparation (Sigma, USA) was used after additional purification and depolymerization (molecular mass of $0.3-0.7) \times 10^{6} \mathrm{Da}$.

Ds poly(I)xpoly(C) preparation (Sigma, USA, lot 023K 4032) was used without additional purification.

Poly (ethylene glycol) (PEG; Serva, Germany; molecular mass 4,000 Da) and cyanine dye SYBR Green I (SG; Sigma, USA) were used without extra purification. SG concentration in solutions was measured spectrophotometrically [18]. SG stock solution was stored at $4^{\circ} \mathrm{C}$ in dark.

Water-salt solutions of DNA, PEG and $\mathrm{NaCl}$ were made in phosphate buffer $(\mathrm{pH} \sim 7.0)$ and then filtered through membrane filters (Millipore, USA) with pore diameter $0.8 \mu \mathrm{m}$ to remove possible mechanical impurities.

Schemes of control tests ( 1 and 2 ) and performed experiments are shown below:

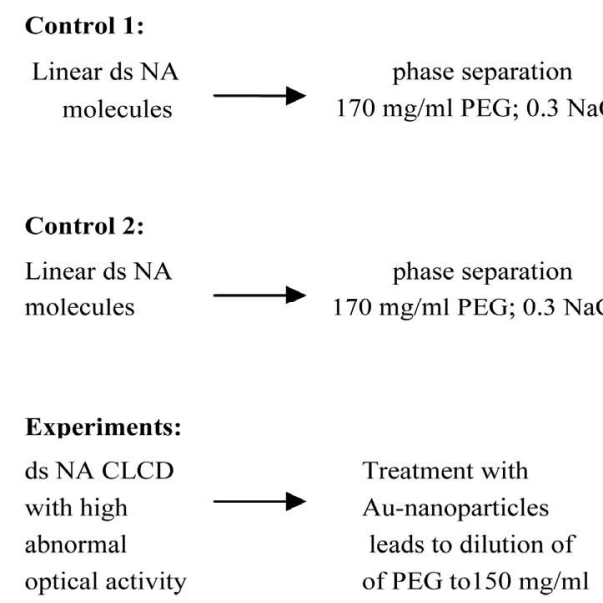

The method of preparation of control cholesteric liquidcrystalline dispersions (CLCDs) of nucleic acids (NA) (control 1) in PEG-containing water-salt solutions is described in [11]. This method was used for preparation of dispersions and phases of ds NA CLCD treated with Aunanoparticles (see "Experiments" above).

Absorbance spectra were taken by spectrophotometer Cary 100 Scan (Varian, USA); and the CD spectra, using portable dichrometer CDS-2 (produced by Institute of
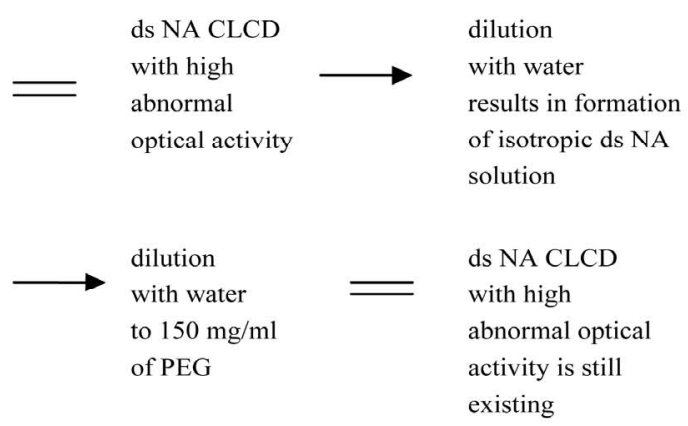

Methods used to study properties of treated ds NA CLCDs: CD-, OD-spectroscopy; confocal microscopy; AFM; SAXS

spectroscopy of the RAS, Troizk, Moscow region) [19].

The fluorescence "images" of particles of the ds DNA CLCD treated with Au nanoparticles were obtained by the confocal microscope "Leica TCS SP5".

The morphology of the ds DNA CLCD particles formed in PEG-containing solutions and treated with Aunanoparticles was examined by AFM. These solutions were filtered through a poly(ethylenetherephtalate) (PETP) nuclear membrane filter with size of pores of $150 \mathrm{~nm}$ 
(produced by the Institute of Crystallography of the RAS). This procedure allows one to immobilize particles on the surface of the filter. Then the filters, containing obtained samples, were dried in air for no less than $1 \mathrm{~h}$. The samples were measured on a scanning probe microscope SmartSPM (produced by AIST-NT Co, Zelenograd, Moscow region, Russia) in semicontact mode. The fpN10 silicon probe with resonance frequency around $250 \mathrm{kHz}$ was used. The initial amplitude of the probe was fixed at $100 \mathrm{~nm}$. The scanning frequency was varied from 2 to $10 \mathrm{~Hz}$; the number of pixels was $256 \times 256$ and $512 \times 512$.

Pellets $(\sim 3 \mathrm{mg})$ obtained after low speed centrifugation of the DNA CLCD treated with Au nanoparticles, were investigated by SAXS. SAXS measurements were performed on the diffractometer AMUR-K (developed at the Institute of Crystallography of the RAS, Moscow) [20]. Technical details of SAXS experiments are described in [20,21]. The analysis of characteristic Bragg peaks on the small-angle scattering curves was made by the program PEAK [22].

\section{Results and Discussion}

The CD spectra of ds DNA and ds poly(I)xpoly(C) CLCDs treated with Au-nanoparticles (diameter $\sim 2 \mathrm{~nm}$ ) are compared in Figure 1.

First of all, one can see that the formation of the liquidcrystalline ds NA dispersions (compare curve 1 and $1^{\mathrm{I}}$ ) induced by the high concentration of PEG in water-salt solution is accompanied by an appearance of intense (abnormal) bands in the CD spectra located in the region of absorption of NA nitrogen bases $\lambda \sim 270 \mathrm{~nm})$. According to the theory $[11,13]$, the appearance of these abnormal (negative or positive) bands unequivocally testify the macroscopic, cholesteric, twist of neighboring NA molecules in particles of dispersion formed as a result of their phase exclusion from PEG-containing watersalt solutions. The negative sign of the band in the CD spectrum proves the left-handed cholesteric twist of the right-handed DNA molecules (B-form, curve 1), whereas the positive sign corresponds the right-handed cholesteric twist of the right-handed poly(I)xpoly(C) molecules (A-form, curve $1^{\mathrm{I}}$ ) in the formed particles. Hence, the abnormal bands in the CD spectrum (Figure 1) located in the absorption region of the nitrogen bases of the NA molecules is the direct evidence for the formation of the particles characterized by helically twisted spatial structure [18-20], or a so-called cholesteric structure of particles and the term CLCD (cholesteric liquid-crystalline dispersion) was used to signify these particles.

In the physicochemical sense, the systems under investigation are PCLCD of the ds NA molecules that are distributed isotropically in the water-salt solution of PEG.

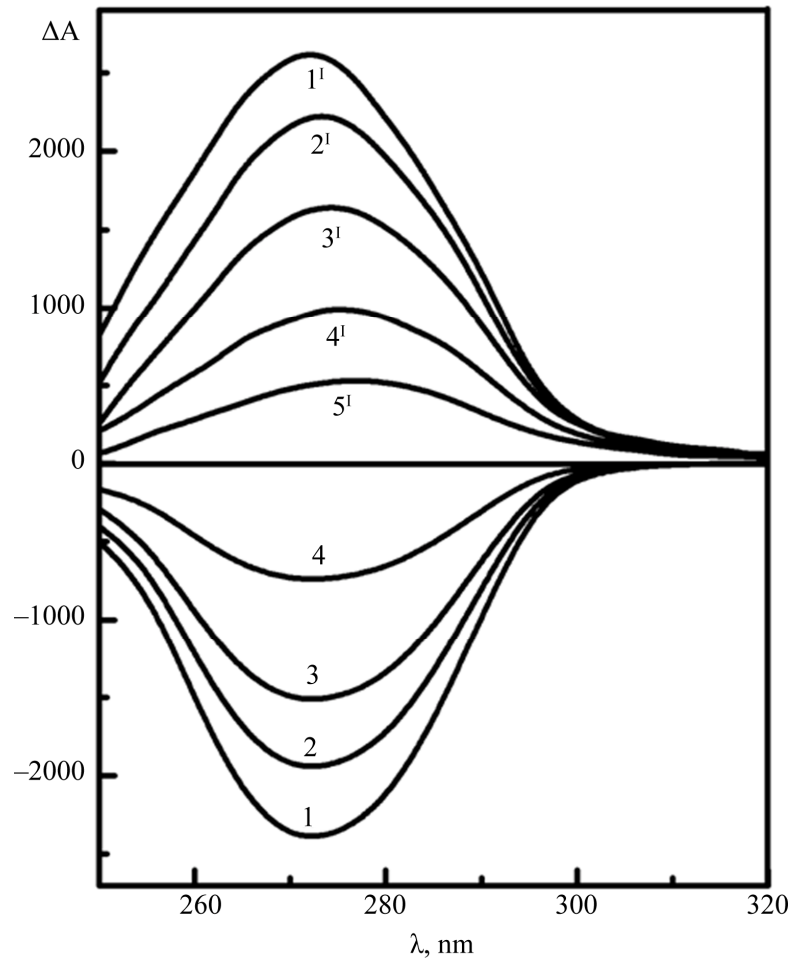

Figure 1. The CD spectra of ds DNA CLCD (curves 1-4) and ds poly(I)xpoly(C) CLCD (curves $1^{\mathrm{I}}-5^{\mathrm{I}}$ ) treated with Au-nanoparticles (2 nm). 1: $C_{\text {nano-Au }}=0 ; 2: C_{\text {nano-Au }}=0.07 \times$ $10^{14}$ particle $/ \mathrm{ml} ; 3: C_{\text {nano-Au }}=0.26 \times 10^{14}$ particle $/ \mathrm{ml} ; 4:$ $\mathrm{C}_{\text {nano-Au }}=0.82 \times 10^{14}$ particle $/ \mathrm{ml} ; \mathrm{C}_{\mathrm{DNA}}=9 \mu \mathrm{g} / \mathrm{ml} ; \mathrm{C}_{\mathrm{PEG}}=$ $150 \mathrm{mg} / \mathrm{ml} ; 0.27 \mathrm{M} \mathrm{NaCl}+1.78 \times 10^{-3} \mathrm{M}$ Na-phosphate buffer. $1^{\mathrm{I}}: \mathrm{C}_{\text {nano-Au }}=0$; $2^{\mathrm{I}}: \mathrm{C}_{\text {nano-Au }}=0.16 \times 10^{14}$ particle $/ \mathrm{ml}$; $3^{\mathrm{I}}: \mathrm{C}_{\text {nano-Au }}=0.33 \times 10^{14}$ particle/ml; $4^{\mathrm{I}}: \mathrm{C}_{\text {nano-Au }}=0.66 \times$ $10^{14}$ particle $/ \mathrm{ml} ; 5^{\mathrm{I}}: \mathrm{C}_{\text {nano-Au }}=0.82 \times 10^{14}$ particle $/ \mathrm{ml}$, $\mathrm{C}_{\text {poly(I)xpoly(C) }}=9 \mu \mathrm{g} / \mathrm{ml}, \mathrm{C}_{\mathrm{PEG}}=170 \mathrm{mg} / \mathrm{ml} ; 0.27 \mathrm{M} \mathrm{NaCl}+$ $1.78 \times 10^{-3} M$ Na-phosphate buffer. $\Delta A=\left(A_{L}-A_{R}\right) \times 10^{-6}$ optical units; $L=1 \mathrm{~cm} ; T=22^{\circ} \mathrm{C}$. In all cases, the $C D$ spectra were taken after $3 \mathrm{~h}$ of treatment.

These particles do not exist in the absence of high osmotic pressure $\left(\pi\right.$, dyne $\left./ \mathrm{cm}^{2}\right)$ of the solvent (for instance, in the water-salt solution, where $\lg \pi$ value is about 6 ) [11]. Note that the particles of the low-molecular-mass ds NA dispersions are "microscopic droplets of concentrated NA solutions", and due to "liquid" mode of packing these particles cannot be "taken in hand" or "directly seen". (The estimations have shown that in the case of ds DNA molecules every PCLCD has a mean diameter about $500 \mathrm{~nm}$; it contains about $10^{4} \mathrm{ds}$ DNA molecules fixed on distances within $2.5-5.0 \mathrm{~nm}$ (depending on osmotic pressure of the solvent $[11,12])$.

Figure 1 shows that in both cases the amplitudes of abnormal bands in the CD spectra drop. The higher the concentration of Au-nanoparticles in solution, the greater is decrease in abnormal band in CD spectra of CLCDs of both types of nucleic acids. 
Figure 1 needs a few comments. First of all, the amplitude of the abnormal band in the CD spectrum depends mainly on helical twisting of quasinematic layers of NA in cholesteric structure of PCLCD $[11,13]$

Secondly, the theory [23] predicts that the change in the efficiency of interaction between ds NA molecules in quasinematic layers must be accompanied by changes in the parameters of the spatial helical structure of CLCD particles and in the value of amplitude of an abnormal band in the CD spectra. This means that the decrease in the amplitudes of abnormal bands in the CD spectra (curves $2-4$ and $2^{\mathrm{I}}-5^{\mathrm{I}}$ ) is connected with the change in the extent of helical twisting of quasinematic layers in structure of PCLCD formed by any type of ds NA molecules, i.e., B-family for DNA and A-family for poly(I) xpoly $(\mathrm{C})$. This effect reflects only one process, i.e., an incorporation of Au-nanoparticles into PCLCD formed by ds NA molecules (Figure 1).

Thirdly, any possible aggregation of independent Aunanoparticles outside of PCLCD can not induce the change in the real value of the abnormal band in the $\mathrm{CD}$ spectra in the region of absorption of NA molecules.

Finally, the changes in amplitudes of abnormal bands in the $\mathrm{CD}$ spectra represent a unique criterion for incorporation of Au-nanoparticles in quasinemtic layers formed by ds NA molecules in PCLCD.

The efficiency of decrease in the amplitudes of abnormal bands for the CLCDs formed by ds DNA or poly (I)xpoly(C) molecules (Figure 1) depends on the size of $\mathrm{Au}$-nanoparticles and the time of treatment with $\mathrm{Au}-$ nanoparticles. For instance, if the diameter of the Aunanoparticles is $2 \mathrm{~nm}$, abnormal band amplitude decreases by $75 \%$ after 4 hours of treatment, but in the case of $15 \mathrm{~nm}$ Au-nanoparticles, it decreases only by $20 \%$ [21]. This fact allows one to suggest that the incorporation of Au-nanoparticles in the content of PCLCD is determined by the distance between ds NA molecules packed in the quasinematic layers under used conditions (150 mg/ml of PEG) [11] (compare Control 2 and Experiments above). Au-nanoparticles with the size about 2 $\mathrm{nm}$ are capable of diffusing between ds NA molecules, because this size is close enough to the distance between ds NA molecules under used conditions (concentration of PEG, etc.) [21]. However, Au-nanoparticles with the sizes equal to $5 \mathrm{~nm}$ and $15 \mathrm{~nm}$ are too "big" in comparison to the distance between ds NA molecules in quasinematic layers, hence these particles do not diffuse effectively into content of ds NA PCLCD.

Explaining the results shown in Figure 1 one can take into account the following.

The first experiments [24,25] demonstrated that $\mathrm{Au}-$ nanoparticles can be assembled near DNA molecules forming supramolecular structures. Later it was shown that an assembly of the Au-nanoparticles is accompanied by formation of planar superstructure consisting of repetitive neighboring linear DNA molecules and $\mathrm{Au}-$ nanopartices. These results unequivocally show that linear, rigid, ds DNA molecules after interaction with $\mathrm{Au}-$ nanoparticles form planar superstructures of type (...Au-DNA-Au-DNA-Au-DNA-Au-...), despite the anisotropic properties of initial ds DNA molecules [4, 26-29].

Hence, on one hand, Au-nanoparticles enforce ds DNA molecules to be organized into planar superstructures, in which neighboring DNA molecules are not only closely packed, but collaterally located.

On the other hand, it is well known that neighboring ds DNA molecules packed in quasinematic layers of PCLCD are helically twisted due to the anisotropic properties of these molecules [11].

Comparison of these two circumstances means that immobilization (incorporation) of Au-nanoparticles in ds NA quasinematic layers will, indeed, results in untwisting of neighboring ds NA layers. This explains the changes in the CD spectra in Figure 1. Indeed, the steric limitations resulting from both dense packing of ds NA molecules in quasinematic layers and fixed PEG concentration in solution prevent the spatial separation (denaturation) of the two strands of the neighboring ds NA molecules [11]. In this case both the incorporation of Aunanoparticles into quasinematic layers of PCLCD and their ordering near ds NA surfaces must be "transformed" by this or that way into the changes in the energy of interaction between neighboring NA molecules in quasinematic layers. Hence, Au-nanoparticles can induce a transition from cholesteric structure of ds NA PCLCD that has high abnormal optical activity to a certain structure, having very low (if any!) abnormal optical activity. For this process the differences in the secondary structure of various ds NA molecules, are not important.

This means that the changes in the $\mathrm{CD}$ spectra (Figure 1) clearly demonstrate that Au-nanoparticles are incorporated in the structure of PCLCD. These changes in the $\mathrm{CD}$ spectra depend only on presence of Au-nanoparticles in the content of quasinematic layers of the PCLCD. The concrete peculiarities of the mechanism of interaction of Au-nanoparticles with ds NA molecules are not important for this effect.

Additional study of visible absorption spectra of Aunanoparticles opens a way to evaluate the size of the assemblies formed by these particles under various conditions [30-37].

Figure 2 shows that the pronounced changes in the visible optical spectrum were observed at the treatment of the ds DNA CLCD with Au-nanoparticles. (The similar results for ds poly(I)xpoly(C) are not shown).

First of all, this treatment is accompanied by an appea- 


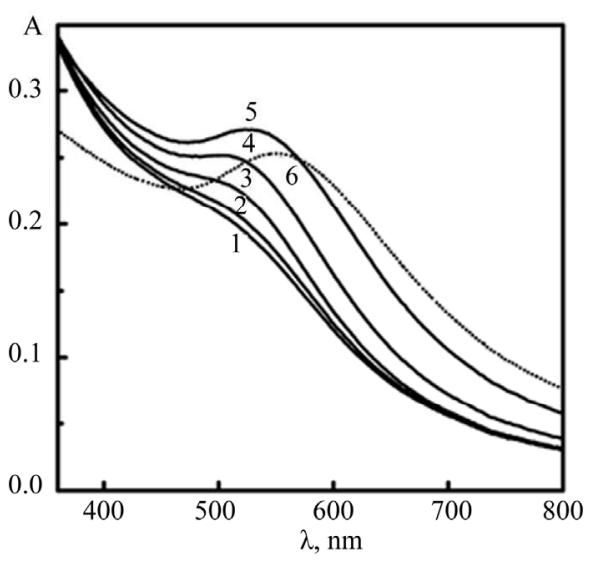

(a)

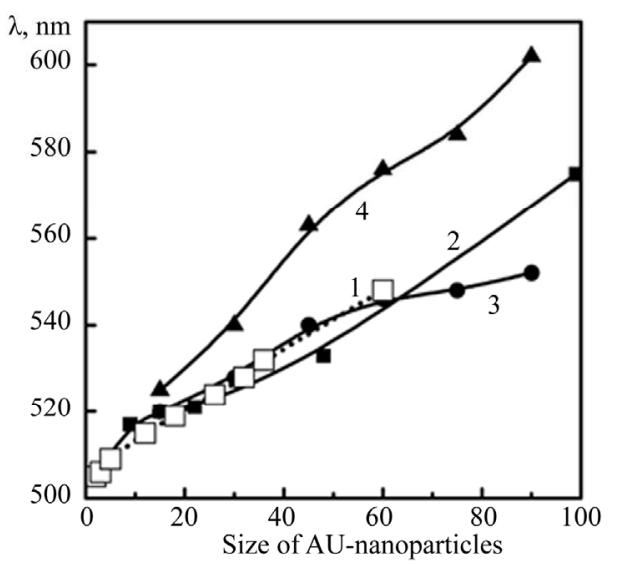

(b)

Figure 2. (a) The change in the absorption spectrum of Au-nanoparticles $(2 \mathrm{~nm})$ at their interaction with ds DNA CLCD. 1: 5 min; 2: 12 min; 3: 43 min; 4: 109 min; $5: 230 \mathrm{~min}$; 6: $1200 \mathrm{~min}$ after the addition of Au-nanoparticles. $C_{D N A}=9 \mu \mathrm{g} / \mathrm{ml} ; C_{P E G}=$ $150 \mathrm{mg} / \mathrm{ml} ; 0.27 \mathrm{M} \mathrm{NaCl}+1.78 \times 10^{-3} \mathrm{M}$ Na-phosphate buffer; $\mathrm{C}_{\text {nano-Au }}=0.82 \times 10^{14}$ particle/ml; $\mathrm{T}=22^{\circ} \mathrm{C}$; (b) The correlation between the position of SPR band and size of Au-nanoparticles. Curve 1-open squares-experimental data from Figure 2(a); curve 2: filled squares [32]; curve 3: filled circles [36]; curve 4: triangles [37].

rance of absorption at $\lambda>505 \mathrm{~nm}$ (surface plasmon resonance (SPR) absorption band) [30,32]. The plasmon resonance is responsible for the pile-violet color of solution containing ds DNA CLCD treated with Au-nanoparticles. This resonance is definitely absent in the initial DNA solution and only slightly defined at $\lambda \sim 505 \mathrm{~nm}$ in colloid solution containing Au-nanoparticles with the size about $2 \mathrm{~nm}$. This means that the immobilization of Au-nanoparticles in the content of ds DNA CLCD particles (Figure 2) brings Au-nanoparticles close together. This results in modifying their local environment and changing the position of the SPR band, because the SPR absorbance is sensitive to local environment [35]. Secondly, the intensity of the SPR band is gradually increased and the maximum of this band is shifted from $\lambda$ $\sim 505 \mathrm{~nm}$ to $\lambda \sim 550 \mathrm{~nm}$ with time.

Comparison of our data (Figure 2(b), curve 1) to available numerical data [30-34,36,37], concerning the correlation between position of the maximum of the SPR band and the size of Au-nanoparticles shows that in our case the change in the SPR band reflects the increase in the size of Au-nanoassemblies from 2 to about $50 \mathrm{~nm}$. Of course, this is a very simple estimation, that does not take into account such factors as real shape of Au-assemblies, local dielectric constant or local refractive index [38].

The comparison of the SPR band typical for PEG containing water-salt solutions added with $5 \mathrm{~nm}$ Au-nanoparticles to analogous solution containing PCLCD formed by ds DNA molecules showed no changes in the SPR band amplitude. The absence of changes in the SPR band was detected in the case of PCLCD treated with 15 $\mathrm{nm}$ Au-nanoparticles as well (data are not shown). These results confirm once again that "big" Au-nanoparticles do not incorporate into the structure of quasinematic layers of PCLCD.

Here one can add that due to the spherical symmetry of individual Au-nanoparticles used by us the formation of assemblies from these particles is a significant problem. But, Figure 2 shows that the ds DNA molecules, ordered in the quasinematic layers, provide, as templates, the necessary symmetry-breaking mechanism to form extended linear structure from Au-nanoparticle fixed between neighboring ds DNA molecules. Finally, after 20 hours one can detect the formation of dark-violet precipitate in the tested solution. The intensive stirring of the solution containing the pellet permits to restore its optical properties and to measure its optical spectrum. Curve 6 in Figure 2 demonstrates the presence of plasmon resonance band located at $\lambda \sim 545-550 \mathrm{~nm}$. Close similarities between the shapes and position of the SPR bands (curves 6 and 5) show that the dark-violet precipitate consists of isolated ds DNA PCLCD, which, in its turn, contain extended structures (about $50 \mathrm{~nm}$ ) formed by Au-nanoparticles. This result shows that the spectral changes in the visible optical spectrum are induced by the processes that take place, mainly, within isolated ds NA PCLCD treated with Au-nanoparticles, but not between neighboring PCLCD.

(The curves of the diminishing in the amplitudes of abnormal bands in the CD-spectra and the increase in the SPR bands for ds DNA and poly(I)xpoly(C) CLCDs treated with Au-nanoparticles showed that there are two different processes: i) quick drop in the amplitudes of the $\mathrm{CD}$ bands due to incorporation of Au-nanoparticles into quasinematic layers of ds NA PCLCD, and ii) relatively 
slow shift of the SPR band due to assembling of neighboring Au-nanoparticles (formation of Au-clusters) in the content of ds NA PCLCD. These two independent processes start simultaneously but differ in their velocities).

Hence, the comparison of Figure 1 to Figure 2 speaks in favor not only incorporation of Au-nanoparticles, but formation of Au-clusters, growing with time [30-34], in the content of ds NA PCLCD.

At fixed osmotic pressure and ionic strength of a PEGcontaining solution the formation of insoluble pellet (see above) shows that Au-clusters, located in quasinematic layers and interacting (independently on mechanism) by this or that way with ds NA molecules, results in the decrease in the solubility of these molecules. In this case the transition of whole PCLCD into insoluble ("rigid") state takes place. Besides, the formation of a multi-layer sandwich-like structure consisting of alternate Au-clusters located both between template ds DNA molecules and ds DNA molecules in neighboring qusinematic layers would lead to an additional contribution to the energy of stabilization of these "rigid" PCLCD [27,39].

The transition of PCLCD into a "rigid" (insoluble) state takes place at definite concentration of Au-clusters. To check this we have used a scheme from "Materials and Methods" (see Controls 1, 2 and Experiment). It is known [11] that ds DNA CLCD exists only at high osmotic pressure of solvent determined by high concentration of PEG. Hence, the dilution of ds DNA CLCD formed in PEG-containing solution with water solution to concentration of PEG much below "critical" value is accompanied by disintegration of the ds DNA PCLCD and formation of ds DNA isotropic solution. This process is usually accompanied by sharp decrease in the amplitude of an abnormal band in the CD spectrum. However, if the structure of PCLCD is stabilized, as a result of interaction with Au-nanoparticles, and can exist even at very low osmotic pressure of the solution, dilution process does not accompanied by disintegration of the structure of ds DNA PCLCD. In this case the disappearance of an abnormal band in the $\mathrm{CD}$ spectrum does not take place.

Figure 3 demonstrates the results of dilution of ds DNA CLCD treated with different concentration of Aunanoparticles (size about $2 \mathrm{~nm}$ ). One can see that at low $\left(\leq 0.26 \times 10^{14}\right.$ particles per ml) concentration of Au-particles added to ds DNA CLCD the process of its dilution is accompanied by disintegration of PCLCD, by formation of isotropic ds DNA solution and, hence, by disappearance of an abnormal band in the CD spectra. However, since "critical" ( $\left.\mathbf{C}^{\mathrm{cr}}{ }_{\text {Nano-Au }}\right)$ concentration of Aunanoparticles about $0.26 \times 10^{14}$ particles per $\mathrm{ml}$, twotimes dilution leads only to twice decrease in concentration of PCLCD and, hence, only in two-fold decrease in

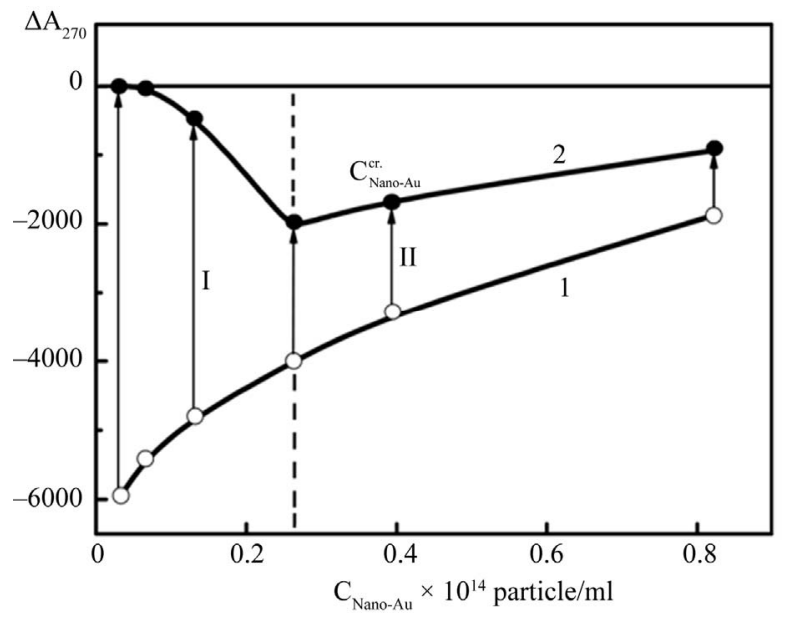

Figure 3. The dependences of the $\Delta A_{270}$-values in the $C D$ spectra for ds DNA CLCDs upon concentration of added Au-nanoparticles (2 nm; curve 1) and for these dispersions treated with Au-nanoparticles and then 2-times diluted with water (curve 2). 1: $\mathrm{C}_{\mathrm{DNA}}=27 \mu \mathrm{g} / \mathrm{ml} ; \mathrm{C}_{\mathrm{PEG}}=150 \mathrm{mg} / \mathrm{ml} ; 0.27$ $\mathrm{M} \mathrm{NaCl}+1.78 \times 10^{-3} \mathrm{M}$ Na-phosphate buffer. 2: $\mathrm{C}_{\mathrm{DNA}}=13$ $\mu \mathrm{g} / \mathrm{ml} ; \mathrm{C}_{\text {PEG }}=75 \mathrm{mg} / \mathrm{ml} ; 0.13 \mathrm{M} \mathrm{NaCl}+0.89 \times 10^{-3} \mathrm{M}$ Na-phosphate buffer. $\Delta A_{270} \times 10^{-6}$ optical units; $L=1 \mathrm{~cm} ; T$ $=22^{\circ} \mathrm{C}$. $\mathrm{C}^{\mathrm{Cr}}$. ${ }_{\text {Nano-Au }}$ the "critical" concentration of the Aunanoparticles. In area "I" the dilution of ds DNA CLCD is accompanied by full disintegration of particles, and final CD amplitude tends to zero; in area "II" the dilution results only in decrease in total concentration of PCLCD, and final CD amplitudes are equal every time to one half of initial values.

the amplitude of an abnormal band (compare curves 1 and 2). The molecular circular dichroism (expressed as $\Delta \varepsilon$ value; $\Delta \varepsilon=\Delta \mathrm{A} / \mathrm{C}_{\mathrm{DNA} \text { PLCD }} \mathrm{x} \mathrm{L}$ ) [11] remains practically constant for these cases. This result unequivocally means that since definite concentration of Au-nanoparticles their interaction with ds DNA molecules provides the conditions necessary for transition of ds DNA CLCD particles into a "rigid" state. (One can add that dilution of ds DNA CLCD treated with big Au-nanoparticles (size of $5 \mathrm{~nm}$ and $15 \mathrm{~nm}$ ) is accompanied under all conditions by full disintegration of PCLCD and by formation of isotropic solutions of ds DNA molecules. The obtained result shows again that "big" Au-nanoparticles do not penetrate into the content of PCLCD and in- teract with ds NA molecules).

Hence, the interaction of Au-nanoparticles $(2 \mathrm{~nm})$ with neighboring ds DNA molecules leads to the formation of a spatially fixed structure of the CLCD. The stability of this structure is determined by the number and properties of Au-clusters rather than the properties of the initial PEG-containing solution. This means that a new structure would persist even in the absence of the high osmotic pressure of the solution. In this case it appears a possibility to investigate the properties of a new-formed 
structure. (Here one can remind again, that a "liquid" mode of spatial packing of ds DNA molecules in the PCLCD prevents their immobilization on the surface of membrane filters. However, if poorly soluble (or insoluble) PCLCD consisting of molecules of the (ds DNA-Au) complexes are formed, the immobilization of these particles on the surface of the nuclear membrane filter becomes possible and the size and shape of these particles can be investigated).

For this purpose, the insoluble PCLCD formed by (ds DNA-Au) complexes were immobilized on the surface of a nuclear membrane filter and the AFM images of these particles were registered. As an example, Figure 4 demonstrates the images (2D and 3D) of ds DNA PCLCD treated with $0.82 \times 10^{14}$ Au-nanoparticles per ml. The shape of the particles is close to elongated spheroids. One can stress that this shape is very similar to the shapes of the rigid PCLCD formed as a result of formation of nanobridges between neighboring ds DNA molecules, or decrease in solubility of ds NA molecules as a result of their treatment with the salt of rare-earth elements [40]. In the shown case, the sizes of particles are varied from $0.1-0.2 \mu \mathrm{m}$ to $0.7 \mu \mathrm{m}$ with $0.4-0.5 \mu \mathrm{m}$ as the average, which is in good agreement with the data on the particle size theoretically calculated for the solutions with a constant osmotic pressure for the initial ds DNA CLCD. This means that the initial size of the PCLCD was not practically changed at interaction of Au-particles with ds DNA.

Figure 4 confirms as well that ds DNA PCLCD treated with Au-particles exist as independent, nonsoluble, objects. The presence of single particles (Figure 4) testifies that at treatment of ds DNA PCLCD by Aunanoparticles the "liquid" character of the DNA packing in these particles is disappeared and the formed particles have a "rigid" spatial structure. Besides, standard sizes of rigid PCLCD formed by (ds DNA-Au) complexes speaks against aggregation of Au-nanoparticles outside PCLCD.

In favor of existence of ds DNA PCLCD in PEGcontaining solutions as independent objects speaks the results obtained with confocal microscope. The ds DNA PCLCD formed in PEG-containing solution $(170 \mathrm{mg} / \mathrm{ml}$ of PEG) was added with Au-nanoparticles and then was additionally treated with dye "SYBR Green". This compound is capable not only of intercalating between nitrogen bases of ds DNA, but also of retaining its high fluorescence, being incorporated even into content of PCLCD formed by ds DNA in PEG-containing solution. As an example, Figure 5 shows the fluorescence "images" of particles of the ds DNA PCLCD treated with $\mathrm{Au}-$ nanoparticles and existing in solution.

These images obtained by the confocal microscope demonstrate that particles of the ds DNA CLCD treated with Au-nanoparticles exist as isolated objects. The mean fluorescence "diameter" of these particles is similar to mean diameter detected for initial ds DNA CLCD particles treated as well with "SYBR Green". This indicates no deformation of the ds DNA secondary structure due to interaction with the Au-nanoparticles. The data shown in Figure 5 are important. They confirm that an appearance of the SPR band is connected with the assembling of Au-nanoparticles realized within individual particles of ds DNA CLCD, in the other words, with formation of Au-clusters between neighboring ds DNA molecules in quasinematic layers of PCLCD.

Taking into account the above results, it was interesting to apply SAXS for investigation of phases formed by ds DNA CLCD treated with Au-nanoparticles.

SAXS permits to probe an internal structure of the object at resolution from about 1 to $100 \mathrm{~nm}$, and can provide valuable structural information both about nanoscale inhomogeneities (particles or clusters) and about
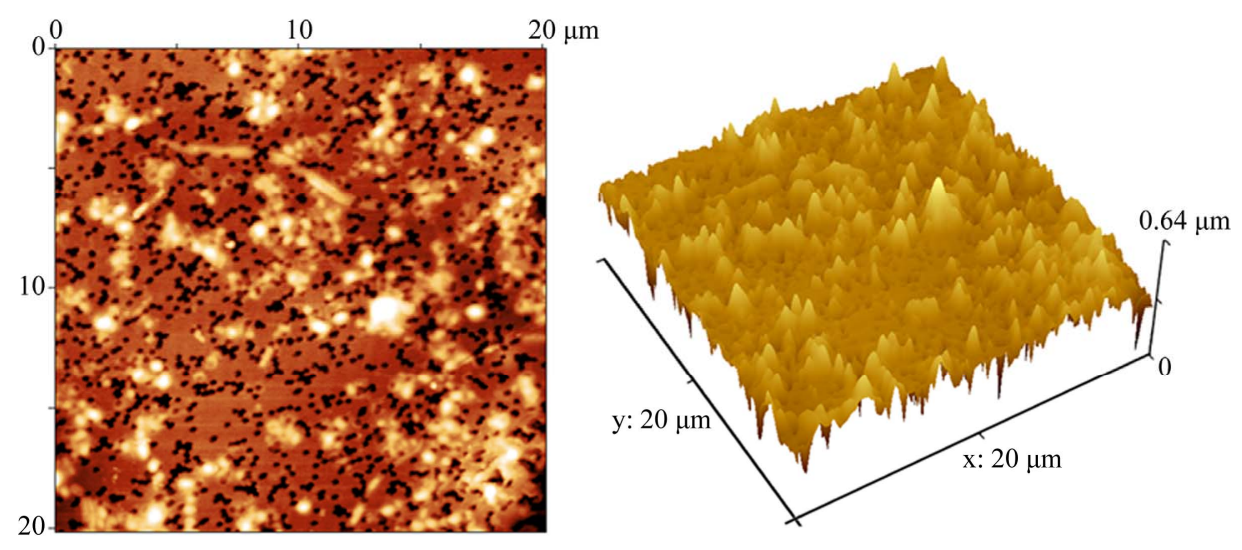

Figure 4. The AFM-images of the ds DNA CLCD particles treated with Au-nanoparticles (2 nm) and immobilized on the surface of a nuclear membrane (PETP) filter (2D: left panel; 3D: right panel). $\mathrm{C}_{\mathrm{Nano}-\mathrm{Au}}=0.82 \times 10^{14}$ particle/ml. (Dark spots are holes in the filter). 


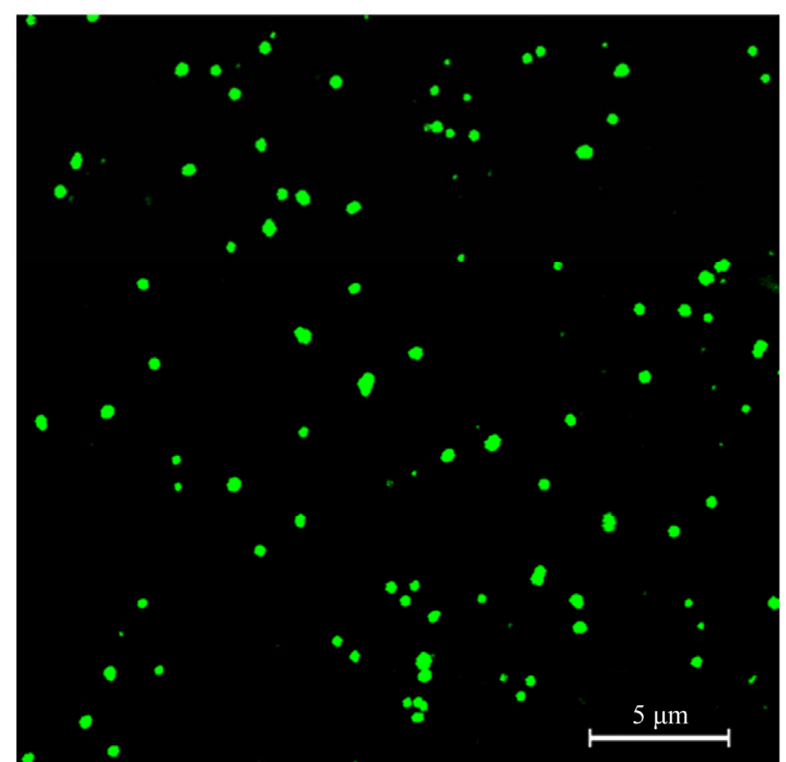

Figure 5. The fuorescence "image" of PCLCD formed by ds DNA in PEG - containing solution and treated with Aunanoparticles $(2 \mathrm{~nm})$ and then with SG. $C_{D N A}=9 \mu \mathrm{g} / \mathrm{ml}$; $\mathrm{C}_{\text {PEG }}=150 \mathrm{mg} / \mathrm{ml} ; 0.27 \mathrm{M} \mathrm{NaCl}+1.78 \times 10^{-3} \mathrm{M}$ Na-phosphate buffer; $\mathrm{C}_{\mathrm{Nano}-\mathrm{Au}}=0.82 \times 10^{14} \mathrm{particle} / \mathrm{ml} ; \mathrm{C}_{\mathrm{SG}}$ $=4.11 \times 10^{-6} \mathrm{M} ; \mathrm{T}=22^{\circ} \mathrm{C}$.

the internal ordering in the sample. The size and shape of the scattering objects can be obtained from the portion of the scattering pattern close to the primary beam (central scattering), while the internal structure is determined using information containing in scattering profiles at higher scattering angles and Bragg peaks [41-44].

Small-angle scattering curves of samples obtained from these phases are shown in Figure 6.

The cholesteric liquid-crystalline phase of initial ds DNA was used as a control sample (curve 1). First of all one can see that samples treated with Au-nanoparticles have diffuse Bragg maxima. Comparison of these curves shows that the interhelical distances $\left(\mathrm{d}_{\text {inter }}\right)$ between adjacent ds DNA molecules in the analyzed samples containing Au-nanoparticles does not increase relatively to Au-free ds DNA CLCD sample. This means that the distance between neighboring quasinematic layers is not changed as a result of incorporation of Au-nanoparticles into structure of ds DNA CLCD. One can say that local concentration of ds DNA molecules remains constant and structural transition due to change in concentration of ds DNA is impossible. Secondly, one can see that the higher Au content in the sample, the lower the amplitude of the Bragg peaks due to their flattening and broadening, which indicates that the ordering is diminished. Taking into account unchanged concentration of ds DNA molecules, this fact shows that the distortion of ordering of ds DNA molecules in neighboring quasinematic layers in

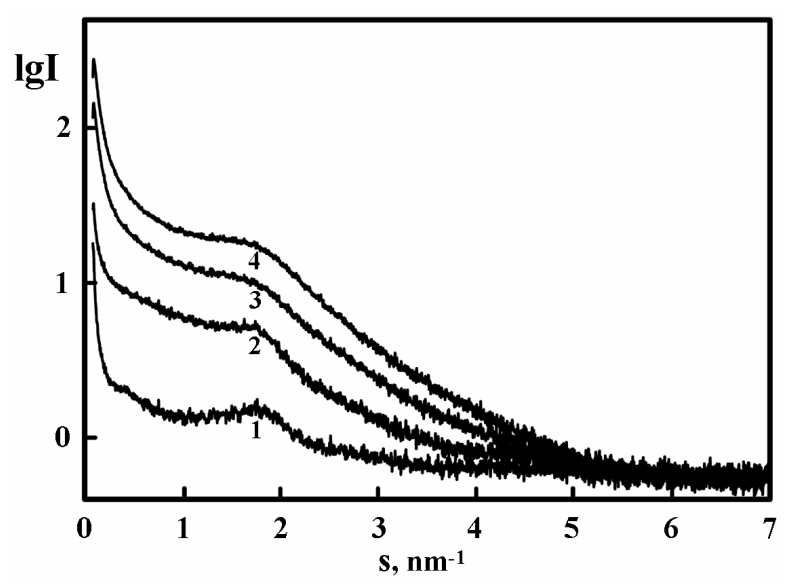

Figure 6. The small-angle $X$-ray scattering curves of the ds DNA CLC phase (curve 1, control) and of the phases containing various concentrations of Au-nanoparticles (curves 2-4). $1-\mathrm{C}_{\text {DNA }}=27 \mu \mathrm{g} / \mathrm{ml}, \mathrm{C}_{\text {PEG }}=150 \mathrm{mg} / \mathrm{ml}, 0.27 \mathrm{M} \mathrm{NaCl}+$ $1.78 \times 10^{-3} \mathrm{M}$ Na-phosphate buffer, $\mathrm{C}_{\text {Nano-Au }}=0 ; 2-\mathrm{C}_{\text {Nano-Au }}$ $=0.07 \times 10^{14}$ particle $/ \mathrm{ml} ; 3-\mathrm{C}_{\text {Nano-Au }}=0.33 \times 10^{14}$ particle/ml; $4-\mathrm{C}_{\mathrm{Nano}-\mathrm{Au}}=0.66 \times 10^{14}$ particle/ml. Solvent, see conditions for curve 1 .

PCLCD is increased. This result correlates with the CD spectra shown in Figure 1, which gives evidence for a decrease in spatial ordering of ds DNA quasinematic layers when concentration of $\mathrm{Au}$ nanoparticles is increased. Hence, one can repeat again that the incorporation of Au-nanoparticles into structure of quasinematic layers of ds DNA PCLCD conditions for collinear location of ds DNA molecules, i.e., this process is accompanied by untwisting of neighboring layers. Finally, samples containing Au-nanoparticles are strongly scattering at very small angles, i.e., in the region of the central scattering, which points to a presence of sufficiently large polydisperse scattering objects (clusters of Au-nanoparticles).

The viewpoint about possible linear arrangement of Au-nanoparticles and the results of size calculation of Au-clusters made by the program GNOM [45] allows one to suggest a hypothetical model reflecting the incorporation of Au-nanoparticles between ds DNA molecules in quasinematic layers (Figure 7).

According to this model, initial Au-nanoparticles have average diameter (size) of about $2 \mathrm{~nm}$. These nanoparticles form linear clusters with an average length $13-15$ $\mathrm{nm}$ and clusters with a maximal length of $\sim 40 \mathrm{~nm}$. Formation of Au-clusters between ds DNA molecules induces violation of mutual orientation of neighboring quasinematic layers. Under these conditions the helical twisting of these layers in the structure of PCLCD is decreased. Thus, the results of SAXS speaks in favor of the statement noted above, according to which the decrease in amplitude of an abnormal band in CD spectrum of ds 


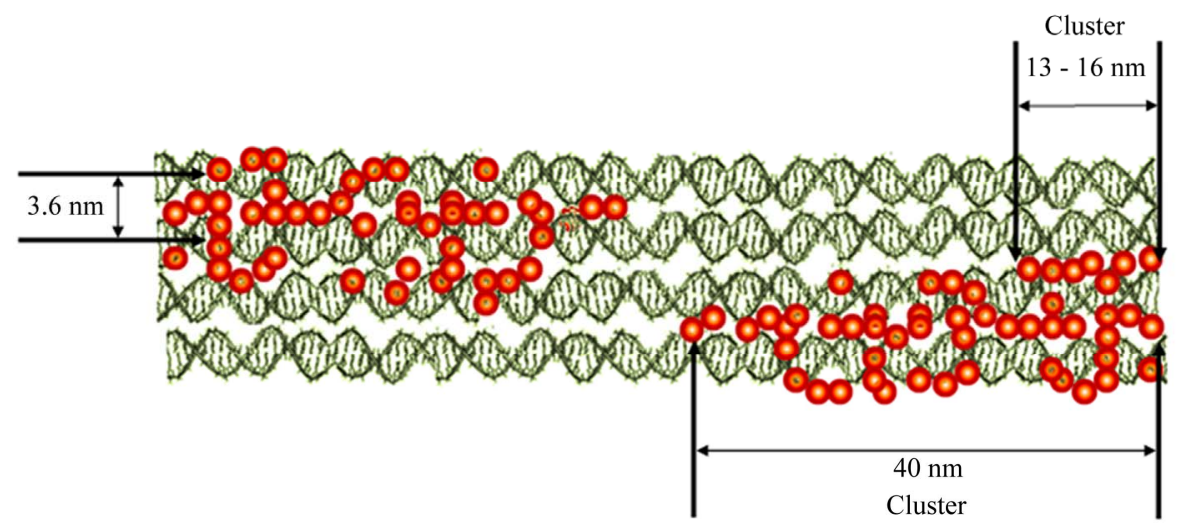

Figure 7. The hypothetical model of location of Au-clusters between ds DNA molecules forming the quasinematic layer in structure of PCLCD.

DNA CLCD treated with Au-nanoparticles reflects the distortion in the arrangement of neighboring quasinematic layers induced by formation of the Au-clusters between them [46]. As a result of this process, spatial structure of PCLCD loses its helical twisting and the transition of type "helically-twisted structure-structure without twisting" is induced. Besides, our calculations showed that the formation of Au-cross-links connecting neighboring ds DNA is possible. Due to the real position of neighboring ds DNA molecules in quasinematic layers, one can expect that in order to connect these molecules, the ends of Au-cross-link should wrap around the ds DNA molecules [29]. It is not excluded, that Au-crosslinks can induce an additional stabilization of the "rigid" structure of ds DNA PCLCD.

Hence, the obtained results demonstrate that interacttion of Au-nanoparticles with ds NA CLCDs results in dual effect: i) it facilitates reorganization of the initial cholesteric structure, and ii) it induces the formation of Au-clusters in particles of ds NA CLCD.

In conclusion, one can add, the effect of introducing $\mathrm{Au}$-nanoparticles into cytoplasm of the cell has been investigated by many scientific teams (see, for instance, $[1,3,7,46])$. However, yet little is known about effect of $\mathrm{Au}$-nanopartricles at the nucleus of the cell. The cell nucleus functions to maintain all processes that occur within the cell, and any disruption within the nucleus would subsequently affect the cell's DNA, thereby disturbing the highly regulated cell cycle. Here can remind that ds DNA CLCD particles reflect some structural and abnormal optical properties of Protozoa chromosomes. High efficiency of interaction of Au-nanoparticles with ds NA CLCDs allows one to suggest that PCLCD formed by ds NA molecules can, indeed, be considered as "molecular sponges", easily "absorbing" nanoparticles of fixed size, interacting by this or that way with ds NA molecules. In the case of Au-nanoparticles such interaction is accom- panied by a strong change in the spatial structure of initial PCLCD of ds NA.

Therefore, the results of this study support the statement, according to which the distortion of mutual orientation of neighboring quasinematic layers formed by ds DNA molecule in spatial chromosome structure, induced by fixation of $\mathrm{Au}$-nanoparticles between fragments of this molecule, can be one of the reasons for genotoxicity of these particles [2,3].

\section{Acknowledgements}

We want to express our thanks to Mikhail N. Savvateev (AIST-NT Co, Zelenograd, Russia) for his technical assistance at the study of the "rigid" PCLCD formed by (DNA-Au) complexes by means of AFM.

\section{REFERENCES}

[1] B. Kang, M. A. Mackey and M. A. El-Sayed, "Nuclear Targeting of Gold Nanoparticles in Cancer Cells Induces DNA Damage, Causing Cytokinesis Arrest and Apoptosis," Journal of the American Chemical Society, Vol. 132, No. 5, 2010, pp. 1517-1519. doi:10.1021/ja9102698

[2] V. Wiwanitkit, A. Sereemaspun and R. Rojanathanes, "Effect of Gold Nanoparticles on Spermatozoa: The First World Report," Fertility \& Sterility, Vol. 91, No. 1, 2009, pp. e7-e8. doi:10.1016/j.fertnstert.2007.08.021

[3] S. T. Zakhidov, T. L. Marshak, E. A. Malolina, A. Yu. Kulibin, I. A. Zelenina, S. M. Pavlyuchenkova, V. M. Rudoy, O. V. Dement'eva, S. G. Skuridin and Y. M. Yevdokimov, "Gold Nanoparticles Impair Nuclear Chromatin Decondensation Process in Murine Sperm Cells in Vitro," Biologicheskie membrany, Russian edition, Vol. 27, No. 4, 2010, pp. 349-353.

[4] Y. P. Liu, W. Meyer-Zaika, S. Franzka, G. Schmid, M. Tsoli and H. Kuhn, "Gold-Cluster Degradation by Transition of B-DNA into A-DNA and Formation of Nanowires," Angewandte Chemie, International Edition, Vol. 42, No. 25, 2003, pp. 2853-2857. doi:10.1002/anie.200250235 
[5] M. Tsoli, H. Kuhn, W. Brandau, H. Esche and G. Schmid, "Cellular Uptake and Toxicity of Au55 Clusters," Small, Vol. 1, No. 8-9, 2005, pp. 841-844. doi:10.1002/smll.200500104

[6] Y. Pan, S. Neuss, A. Leifert, M. Fischer, F. Wen, U. Simon, G. Schmid, W. Brandau and W. Jahnen-Dechent, "Size-Dependent Cytotoxicity of Gold Nanoparticles," Small, Vol. 3, No. 11, 2007, pp. 1941-1949. doi:10.1002/smll.200700378

[7] E. Boisselier and D. Astruc, "Gold Nanoparticles in Nanomedicine: Preparations, Imaging, Diagnostics, Therapies and Toxicity," Chemical Society Reviews, Vol. 38, No. 6, 2009, pp. 1759-1782. doi:10.1039/b806051g

[8] X. D. Zhang, M. L. Guo, H. Y. Wu, Y. Q. Ding, X. Feng and L. A. Zhang, "Irradiation Stability and Cytotoxicity of Gold Nanoparticles for Radiotherapy," International Journal of Nanomedicine, Vol. 4, No. 9, 2009, pp. 165-173.doi:10.2147/IJN.S6723

[9] Y. Jin, M. Wu and X. Zhao, "Toxicity of Nanomaterials to Living Cells," Technical Proceedings of the 2005 NSTI Nanotechnology Conference and Trade Show, Vol. 1, 2005, pp. 274-277.

[10] J. Ai, E. Biazar, M. Jafarpour, M. Montazeri, A. Majdi, S. Aminifard, M. Zafari, H. R. Akbari and H. G. Rad, "Nanotoxicology and Nanoparticle Safety in Biomedical Designs," International Journal of Nanomedicine, Vol. 6, No. 5, 2011, pp. 1117-1127.

[11] Yu. M. Yevdokimov, V. I. Salyanov, S. V. Semenov and S. G. Skuridin, "DNA Liquid-Crystalline Dispersions and Nanostructures," In: Yu. M. Yevdokimov, Ed., CRC Press, Taylor \& Francis group, London, 2011, p. 320

[12] F. Livolant and A. Leforestier, "Condensed Phases of DNA: Structures and Phase Transitions," Progress in Polymer Science, Vol. 21, No. 6, 1996, pp. 1115-1164. doi:10.1016/S0079-6700(96)00016-0

[13] Y. M. Yevdokimov, V. I. Salyanov, S. G. Skuridin, S. V. Semenov and O. N. Kompanets., "The CD Spectra of Doublestranded DNA Liquid-Crystalline Dispersions," Nova Science Publishers, New York, 2011, p 103.

[14] Y. M. Yevdokimov, S. G. Skuridin and B. A. Chernuha, "The Background for Creating Biosensors Based on Nucleic Acid Molecules," In: A. P. F. Turner and Y. M. Yevdokimov, Eds., Advances in Biosensors, JAI Press, London, 1995, pp. 143-164.

[15] J. Turkevich, P. C. Stevenson and J. Hillier, "A Study of the Nucleation and Growth Processes in the Synthesis of Colloidal Gold," Discussions of the Faraday Society, Vol. 11, 1951, pp. 55-75. doi:10.1039/df9511100055

[16] K. R. Brown, D. G. Walter and M. J. Natan, "Seeding of Colloid-Dal Au-nanoparticle Solutions 2. Improved Control of Particle Size and Shape," Chemistry of Materials, Vol. 12, No. 2, 2000, pp. 306-313. doi: $10.1021 / \mathrm{cm} 980065 \mathrm{p}$

[17] D. G. Duff, A. Baiker and P. P. Edwards, "A New Hydrosol of Gold Clusters 1. Formation and Particle Size Variation," Langmuir, Vol. 9, No. 9, 1993, pp. 2301-2309. doi: $10.1021 / 1 \mathrm{a} 00033 \mathrm{a} 010$
[18] H. Zipper, H. Brunner, J. Bernhagen and F. Vitzthum, "Investigations on DNA Intercalation and Surface Binding by SYBR Green I, Its Structure Determination and Methodological Implications," Nucleic Acids Research, Vol. 32, No. 12, 2004, pp. e103. doi:10.1093/nar/gnh101

[19] O. N. Kompanets, "Portable Optical Biosensors for Detection of Biologically Active and Toxic Compounds," Uspekhi Fizicheskikh Nauk, Russian edition, Vol. 174, No. 6, 2004, pp. 686-690.

[20] E. V. Shtykova, V. V. Volkov, V. I. Salyanov and Yu. M. Yevdokimov, "SAXS_Data_Based Structural Modeling of DNA-Gadolinium Complexes Fixed in Particles of Cholesteric Liquid-Crystalline Dispersions," European Biophysics Journal, Vol. 39, No. 9, 2010, pp. 1313-1322. doi:10.1007/s00249-010-0584-0

[21] S. G. Skuridin, V. A. Dubinskaya, E. V. Shtykova, V. V. Volkov, V. M. Rudoy, O. V. Dement'eva, V. A. Kuzmin, E. S. Lisitsyna, S. T. Zakhidov, I. A. Zelenina and Yu. M. Yevdokimov, "Retention of Gold Nanoparticles in the Structure of Quasienematic Layers Formed by DNA Molecules," Biologicheskie membrany, Russian edition, Vol. 28, No. 3, 2011, pp. 191-198.

[22] P. V. Konarev, V. V. Volkov, A. V. Sokolova, M. H. J. Koch and D. I. Svergun, "PRIMUS: A Windows PCBased System for Small-Angle Scattering Data Analysis," Journal of Applied Crystallography, Vol. 36, 2003, No. 5, pp. 1277-1282. doi:10.1107/S0021889803012779

[23] V. A. Belyakov, V. P. Orlov, S. V. Semenov, S. G. Skuridin and Yu. M. Yevdokimov, "Comparison of Calculated and Observed CD Spectra of Liquid Crystalline Dispersions Formed from Double-Stranded DNA and from DNA Complexes with Colored Compounds," Liquid Crystals, Vol. 20, No. 6, 1996, pp. 777-784. doi:10.1080/02678299608033172

[24] C. A. Mirkin, R. L. Letsinger, R. C. Mucic and J. J. Storhoff, "A DNA-Based Method for Rationally Assembling Nanoparticles into Macroscopic Materials," Nature, Vol. 382, No. 6592, 1996, pp. 607-609. doi:10.1038/382607a0

[25] A. P. Alivisatos, K. P. Johnsson, X. Peng, T. E. Wilson, C. J. Loweth, M. P. Bruchez and P. Scultz, "Organization of Nanocrystal Molecules Using DNA,” Nature, Vol. 382, No. 6592, 1996, pp. 609-611. doi:10.1038/382609a0

[26] A. Kumar, M. Pattarkine, M. Bhadbhade, A. B. Mandale, K. N. Ganesh, S. S. Datar, C. V. Dharmadhikari and M. Sastry, "Linear Superclusters of Colloidal Gold Particles by Electrostatic Assembly on DNA Templates," Advanced Materials, Vol. 13, No. 5, 2001, pp. 341-344. doi:10.1002/1521-4095(200103)13:5<341::AID-ADMA3 41>3.0.CO;2-X

[27] M. Sastrya, A. Kumar, S. Datar and C. V. Dharmadhikari, "DNA-Mediated Electrostatic Assembly of Gold Nanoparticles into Linear Arrays by a Simple Drop-Coating Procedure," Applied Physics Letters, Vol. 78, No. 19, 2001, pp. 2943-2945. doi:10.1063/1.1370993

[28] M. G. Warner and J. E. Hutchison, "Linear Assemblies of Nanoparticles Electrostatically Organized on DNA Scaffolds," Nature Materials, Vol. 2, No. 4, 2003, pp. 272 277. doi:10.1038/nmat853 
[29] L. V. Zherenkova, P. V. Komarov and P. G. Khakatur, "Simulation of the Metallization of a Fragment of a DeOxyribonucleic Acid Molecule with Gold Nanoparticles," Colloid Journal, Russian edition, Vol. 69, No. 6, 2007, pp. 753- 765.

[30] S. Link and M. A. El-Sayed, "Spectral Properties and Relaxation Dynamics of Surface Plasmon Electronic Oscillations in Gold and Silver Nanodots and Nanorods," The Journal of Physical Chemistry B, Vol. 103, No. 40, 1999, pp. 8410-8426. doi:10.1021/jp9917648

[31] S. L. Westcott, S. J. Oldenburg, T. R. Le and N. J. Halas, "Construction of Simple Gold Nanoparticle Aggregates with Controlled Plasmon-Plasmon Interactions," Chemical Physics Letters, Vol. 300, No. 5-6, 1999, pp. 651-655. doi:10.1016/S0009-2614(98)01410-9

[32] S. Link and M. A. El-Sayed, "Shape and Size Dependence of Radioactive, Non Radioactive and Photothermal Properties of gold Nanocrystals," International Reviews in Physical Chemistry, Vol. 19, No. 3, 2000, pp. 409-453. doi:10.1080/01442350050034180

[33] K. H. Su, Q. H. Wei, X. Zhang, J. J. Mock, D. R. Smith and S. Schultz, "Interparticle Coupling Effects on Plasmon Resonances of Nanogold Particles," Nano Letters, Vol. 3, No. 8, 2003, pp. 1087-1090. doi:10.1021/n1034197f

[34] W. Rechberger, A. Hohenau, A. Leitner, J. R. Krenn, B. Lamprecht and F. R. Aussenegg, "Optical Properties of Two Interacting Gold Nanoparticles," Optics Communications, Vol. 220, No. 1-3, 2003, pp. 137-141. doi:10.1016/S0030-4018(03)01357-9

[35] P. V. Kamat, "Photophysical, Photochemical and Photocatalytic Aspects of Metal Nanoparticles," The Journal of Physical Chemistry B, Vol. 106, No. 32, 2002, pp. 77297744. doi:10.1021/jp0209289

[36] N. G. Khlebtsov, A. G. Melnikov, L A. Dykman. and V. A Bogatyrev, "Optical Properties and Biomedical Applications of Nanostructures Based on Gold and Silver Bioconjugates. Photopolarimetry in Remote Sensing," In: G. Videen, Ya. S. Yatskiv and M. I. Mishchenko, Eds.,

\section{Abbreviations}

Au nanoparticles: gold nanoparticles,

CD: circular dichroism,

OD: optical density,

CLCD: cholesteric liquid-crystalline dispersion,

Ds: double-stranded,

NA: nucleic acid,

PCLCD: particles of cholesteric liquid-crystalline dispersion,
NATO Science Series, II. Mathematics, Physics, and Chemistry, Vol. 161, 2004, pp. 265-308.

[37] L. Dykman, V. Bogatyrev, S. Shchyogolev and N. Khlebtsov, "Gold Nanoparticles: Synthesis, Properties, Biomedical Applications," Nauka, Russian Edition, Moscow, 2008, pp. 70-78.

[38] P. Mulvaney, "Surface Plasmon Spectroscopy of Nanosized Metal Particles," Langmuir, Vol. 12, No. 3, 1996, pp. 788-800. doi:10.1021/la9502711

[39] G. Decher, "Fuzzy Nanoassemblies: Toward Layered Polymeric Multicomposites," Science, Vol. 277, No. 5330 1997, pp. 1232-1237. doi:10.1126/science.277.5330.1232

[40] Yu. M. Yevdokimov, "From Particles of Liquid Crystalline Dispersions to Rigid Deoxyribonucleic Acid Nanoconstructions," Liquid Crystals Today, Vol. 20, No. 1, 2011, pp. 2-19. doi:10.1080/1358314X.2011.549300

[41] A. Guinier and G. Fournet, "Small-Angle Scattering of X-Rays," John Wiley and Sons, New York, 1955, p. 269.

[42] O. Glatter and O. Kratky, "Small-Angle X-Ray Scattering," Academic Press, London, 1982, p. 515.

[43] L. A. Feigin and D. I. Svergun, "Structure Analysis by Small-Angle X-ray and Neutron Scattering," Plenum Press, New York, 1987.

[44] H. D. Mertens, D. I. Svergun, "Structural Characterization of Proteins and Complexes Using Small-Angle X-ray Solution Scattering," Journal of Structural Biology, Vol. 172, No. 1, 2010, pp. 128-141. doi:10.1016/j.jsb.2010.06.012

[45] D. I. Svergun, A. V. Semenyuk and L. A. Feigin, "Small-Angle-Scattering-Data Treatment by the Regularization Method," Acta Crystallographica, Vol. A44, 1988, pp. 244-250.

[46] A. Rocha, Y. Zhou, S. Kundu, J. M. Gonzalez, S. B. Vinson and H. Liang, "In vivo Observation of Gold Nanoparticles in the Central Nervous System of Blaberus discoidalis," Journal of Nanobiotechnology, Vol. 9, No. 1, 2011. doi:10.1186/1477-3155-9-5

SPR: surface plasmon resonance,

AFM: atomic force microscopy,

SAXS: small-angle X-ray scattering,

SG: SYBR Green I,

PEG: poly(ethylene glycol),

PETP: poly(ethylene therephtalate). 